\title{
A 5.9mW/Gb/s 7Gb/s/pin 8-Lane Single-Ended RX with Crosstalk Cancellation Scheme using a XCTLE and 56-tap XDFE in 32nm SOI CMOS
}

\author{
A. Cevrero ${ }^{1, \S}$, C. Aprile ${ }^{2, \S}$, P.A. Francese ${ }^{1}$, U. Bapst ${ }^{1}$, C. Menolfi ${ }^{1}$, M. Braendli ${ }^{1}$, \\ M. Kossel ${ }^{1}$, T. Morf ${ }^{1}$, L. Kull ${ }^{1}$, H. Yueksel ${ }^{1}$, I. Oezkaya ${ }^{1}$, Y. Leblebici ${ }^{2}$, V. Cevher ${ }^{2}$, and T.Toifl ${ }^{1}$ \\ ${ }^{1}$ IBM Research - Zurich, Rueschlikon, Switzerland, ${ }^{2}$ EPFL, Lausanne, Switzerland \\ $\S$ both authors contributed equally to this work
}

\begin{abstract}
This work reports an 8-lane single-ended RX featuring compact and low power far-end crosstalk (FEXT) cancellation circuits. The RX data-path consists of a cross continuous-time linear equalizer (XCTLE) to remove FEXT by nearest aggressors within the channel bundle. Residual post-cursor FEXT is suppressed by a direct feedback $7 \times 8$-tap cross decisionfeedback equalizer (XDFE). A CTLE and 8-tap DFE equalize single-ended channels with $28 \mathrm{~dB}$ insertion loss at Nyquist frequency without TX FFE. The circuit, fabricated in $32 \mathrm{~nm}$ SOI CMOS, was measured to receive $7 \mathrm{~Gb} / \mathrm{s} /$ pin PRBS11 data at $\mathrm{BER}<10^{-12}$ with $12.5 \% \mathrm{UI}$ margin. It occupies $300 \mathrm{x} 350 \mu \mathrm{m}^{2}$ with an energy efficiency of $5.9 \mathrm{~mW} / \mathrm{Gb} / \mathrm{s}$.

\section{Introduction}

Over the past decade, aggregate I/O bandwidth requirements have increased at a rate of approximately $2 x$-to- $3 x$ every 2 years [1]. Single-ended-signaling improves aggregate datarate, resulting in nearly twice the performance of similar buses operating with two differential lines per signal. Unfortunately, single-ended PCB traces with reduced lane-to-lane spacing suffer from increased crosstalk (xtalk) noise by electromagnetic coupling. A significant challenge is to ensure proper signal transmission over single-ended wires at rates previously attainable only with differential pairs. In this work, a powerful equalization method is proposed that combines a cross continuous-time linear equalizer (XCTLE) and multi-tap cross decision-feedback equalizer (XDFE). Since far-end crosstalk (FEXT) is approximately proportional to the derivative of the channel, $\operatorname{FEXT}(\omega)=-j \omega \beta H(\omega)$ [2] a XCTLE equalizes xtalk by differentiating the received signals from nearest neighbors and adding them with appropriate gain $(\mathrm{G} 0, \mathrm{G} 1)$ to match the xtalk strength $\beta$ as proposed in [2]. Compared to [2], the implemented RX does not require wider spacing between bundle pairs, since residual error terms are suppressed by the XDFE. Furthermore, a XDFE compensates non trivial xtalk patterns generated by connectors and via-arrays. Only the synergy between XCTLE and XDFE results in error free data for the channel investigated in this work. Although RX with multi XDFE taps are commonly used in ADC-based 100/10GBASE$\mathrm{T}$ transceivers, they are not yet used for chip-to-chip link owing to their increased demand for power and area. A low power analog 56-tap XDFE is implemented using a switched capacitor (SC) approach proposed in [3].

\section{Architecture}

Fig. 1 shows the architecture of our RX circuit which is intended for use in source-synchronous links. It consists of 8 single-ended data lanes and 1 shared differential clock lane. The reference voltage $V_{\text {ref }}$ is extracted from the differential clock common-mode using a low-pass filter. The received signal is terminated to $\mathrm{V}_{\mathrm{dd}}=1 \mathrm{~V}(1 \mathrm{~V}, 500 \mathrm{mV}$ DC levels at RXin) using T-coils for bandwidth enhancement in the product-level ESD protection circuit. The signals on the victim and adjacent aggressor lanes are processed by a XCTLE, which uses two single-ended high-pass RC filters to differentiate the aggressor signals. The xtalk cancellation and forward signals are weighted into 3 VGAs to adjust the xtalk cancellation target before being summed. The XCTLE also performs singleended to differential conversion. The xtalk-equalized signal passes into a 2-stage CTLE which provides up to $17 \mathrm{~dB}$ peaking at $3.5 \mathrm{GHz}$ with $-3.7 \mathrm{~dB}$ DC-gain, and the CTLE output is then fed to an integrating amplifier which connects to the 8-tap SC DFE and 7x8-tap SC XDFE, resulting in 64-tap per lane in total. 56 XDFE cells are driven by FIFO data from 7 aggressor lanes. A 1:4 demux outputs quarter-rate data to a digital correlator/PRBS checker for adjusting all RX parameters (latch offsets, DFE and XDFE coefficients, CTLE and XCTLE settings).

Fig. 2 shows the XCTLE circuit diagram. It consists of two passive high-pass $\mathrm{RC}(\mathrm{R}=972 \Omega, \mathrm{C}=30 \mathrm{fF})$ filters for implementing the differentiators. $\mathrm{R}$ and $\mathrm{C}$ values have been chosen such that they provide return-loss below $-10 \mathrm{~dB}$ up to $4 \mathrm{GHz}$ at each of the $50 \Omega$ terminated RX inputs. VGA bias currents are binary weighted with 4-bit resolution. The XCTLE dissipates only $0.56 \mathrm{~mW} / \mathrm{Gb} / \mathrm{s}$. Including CTLE, the analog front-end has an energy efficiency of $1.56 \mathrm{~mW} / \mathrm{Gb} / \mathrm{s}$.

The DFE core is shown in Fig.3. The DFE runs at full rate for improved area efficiency. The continuous time signal equalized by the CTLE is amplified by a current integrating stage for $1 / 2$ UI. The absence of samplers is advantageous as it avoids $\mathrm{kT} / \mathrm{C}$ noise with a cost of $0.9 \mathrm{~dB}$ loss due to $1 / 2 \mathrm{UI}$ timewindow integration. The analog DFE correction is performed by adding charge on the integration node with a digitally programmable SC-DAC. The SC implementation relaxes timing of the DFE loop compared with current summation DFE [3]. Each capacitive DAC has 6-bit resolution with $1 \mathrm{LSB}=250 \mathrm{aF}$ $\left(\mathrm{C}_{\max }=15.75 \mathrm{fF}\right)$ implemented with M1-M2 finger caps. To cover a large correction range, tap 1 uses 3 SC cells connected in parallel. To close the DFE tap-1 timing with reasonable margin the data representation is kept in pre-charged dynamic logic format from the offset-programmable strongARM latch to the input of each SC cell. Each DFE core drives 8 DFE cells and $7 \times 8$ XDFE cells ( 8 cells per victim). Each lane includes an additional offset-programmable latch (error/amplitude sampler) for RX internal eye measurement and DFE tap calibration.

\section{Measurement Results}

The dies were flip-chip mounted on an high-frequency, low loss substrate (LCP) that itself is embedded in a rigid metallic frame including impedance-matched high-frequency coaxial connectors. The RX was connected to a $72 \mathrm{~cm}$ channel bundle (Rogers PCB) with lane-spacing equals to 1.5 times lanewidth $(\mathrm{s}=1.5 \mathrm{w}=142 \mu \mathrm{m})$ which includes 2 daughter boards, 4 $5 \mathrm{~mm}$ thru-via-arrays and 4 Erni MicroSpeed connectors along the signal path to create severe FEXT. The signal loss including cables, connectors and package was about $28 \mathrm{~dB}$ at $3.5 \mathrm{GHz}$, 


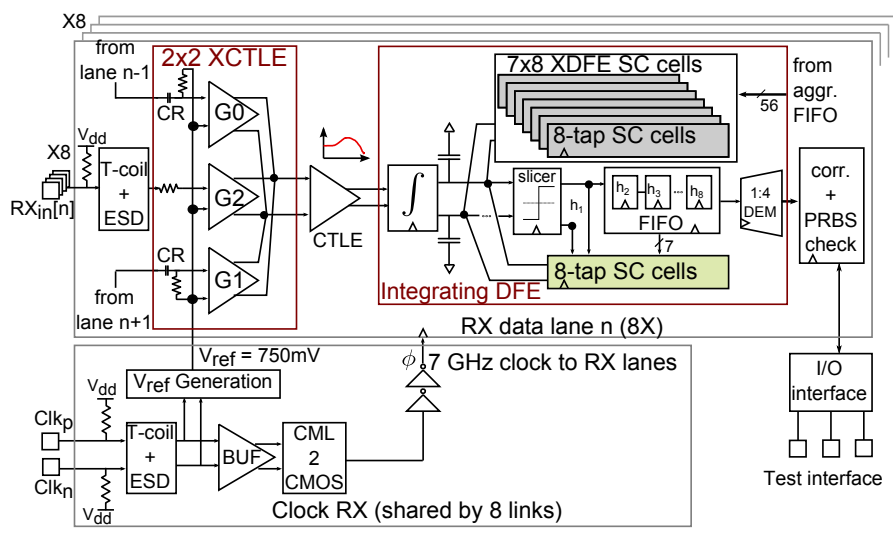

Fig. 1. 8-lane single-ended RX architecture with XDFE and XCTLE.

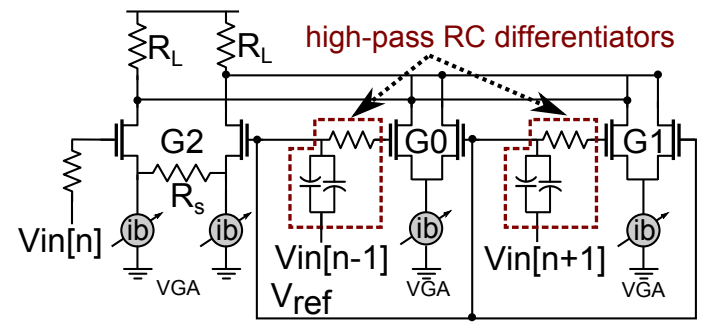

Fig. 2. Proposed XCTLE circuit diagram.

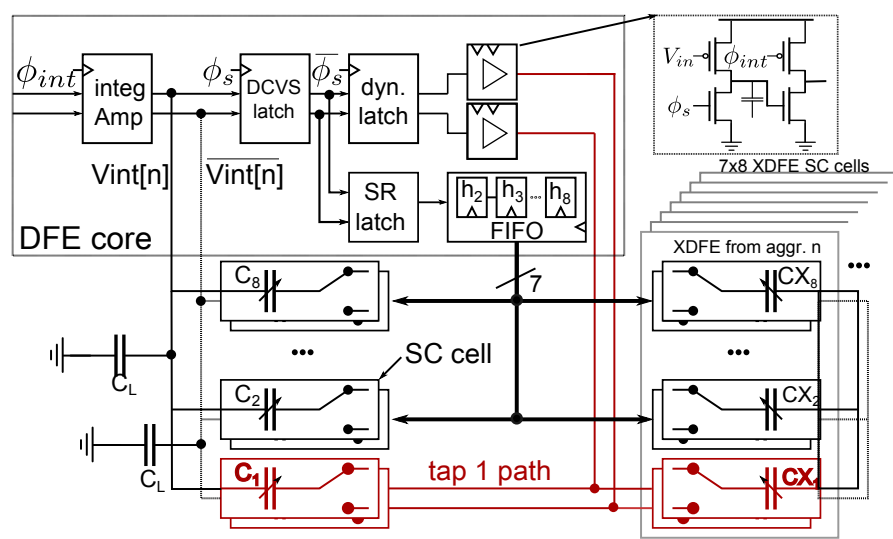

Fig. 3. DFE core with fast tap-1 feedback.

with FEXT from adjacent lanes 4dB lower. A 3-lane measurement was performed owing to limitation of the measurement equipment. Three uncorrelated 7Gb/s NRZ streams (PRBS7 on aggressors, PRBS11 on victim) were sent over 3 adjacent lanes. The correlator/PRBS checker was used to adjust the DFE and XDFE coefficients driving the correlation with postcursor channel taps to zero (Fig. 4 left). The BER bathtub curves are shown in Fig.4 (right). With the aggressor turned off, the RX eye is open with a horizontal margin of $40 \%$ at $10^{-12}$ BER. Once 2 aggressors are switched on, the link no longer operates error free $\left(10^{-4} \mathrm{BER}\right)$. After turning on xtalk cancellation, the eye is reasonably open with a $12.5 \%$ UI margin, showing that both a XCTLE and a XDFE equalizer are necessary to ensure error-free operation of the RX. The vertical eye margins, measured by sweeping the data latch offset and reading out the internal error counter, are $22.4 \mathrm{mV}_{\text {ppdiff }}$ and $64 \mathrm{mV}_{\text {ppdiff }}$ at $10^{-8} \mathrm{BER}$ with and without xtalk, respectively. The internal data eyes displayed in Fig.5 were generated by sweeping the data horizontally with an Agilent phase generator and vertically by sweeping the amplitude programmable latch offset with an R2R voltage DAC. The measured power efficiency of the RX is $5.9 \mathrm{~mW} / \mathrm{Gb} / \mathrm{s}$ with $1 \mathrm{~V}$ supply at package.

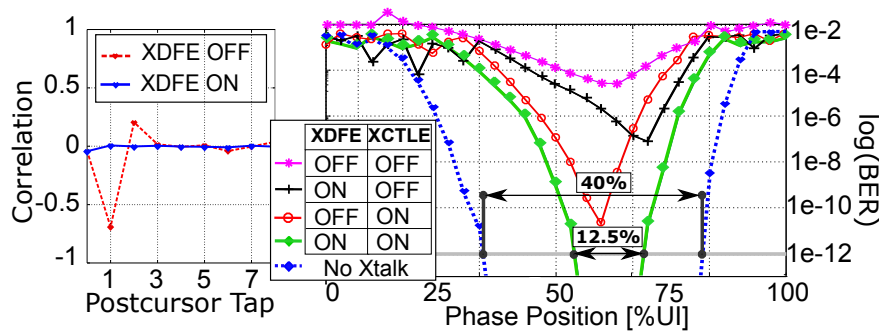

Fig. 4. Measured bathtub plots (right) and correlation with postcursor taps with and without XDFE (left).
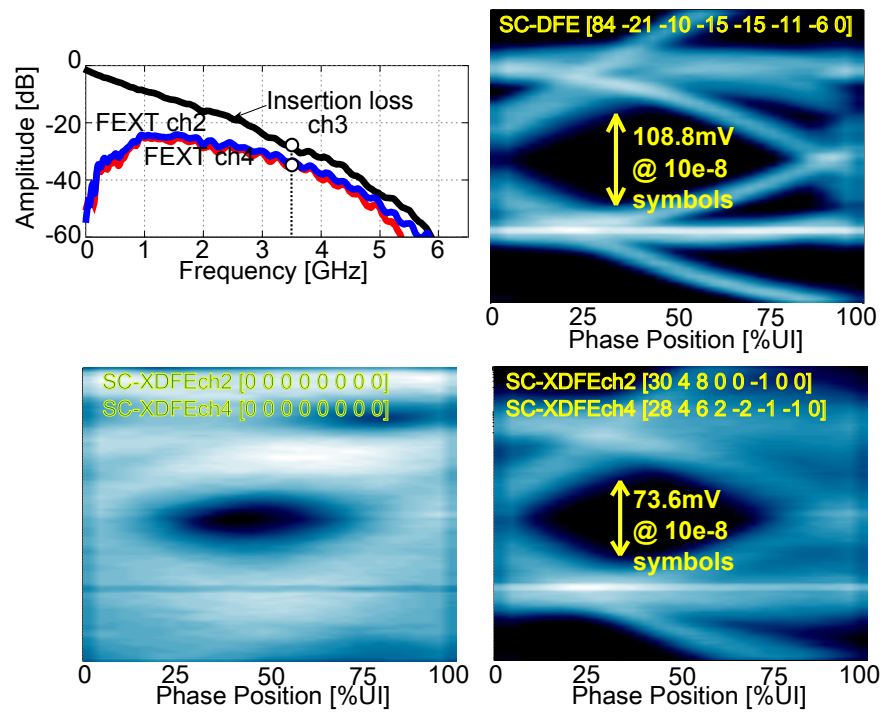

Fig. 5. Channel attenuation (top left) and received eye diagrams with silent aggressors (top right), xtalk cancellation off (bottom left), xtalk cancellation on (bottom right). The programmed SC DAC code is also shown.

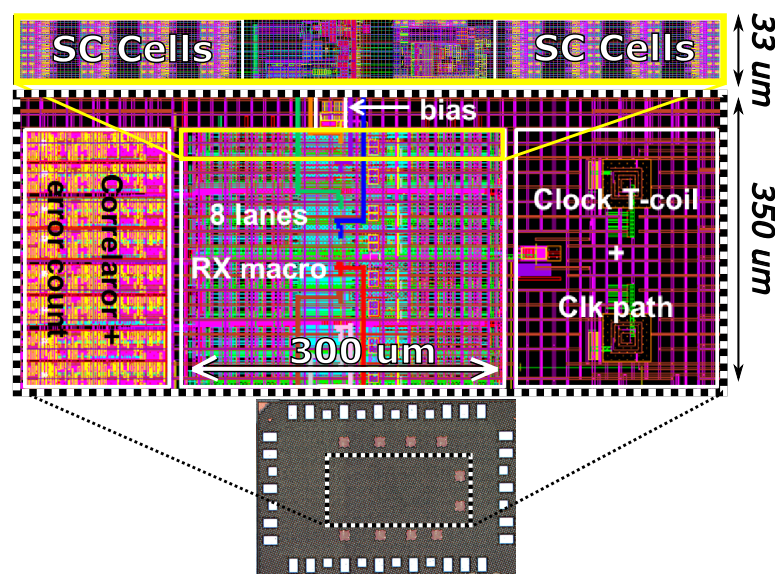

Fig. 6. Layout of RX and die photograph.

The layout of the fabricated circuit, whose RX macro measures 300x350 $\mu \mathrm{m}^{2}$ is shown in Fig.6.

\section{Acknowledgment}

This work was supported in part by the European Commission under grant ERC Future Proof and by the Swiss Science Foundation under grants SNF 200021-146750 and SNF CRSII2-147633.

\section{References}

[1] "ISSCC 2014 technology trends," [Online].

[2] T. Oh, et al., " $4 \times 12$ Gb/s 0.96 pJ/b/lane analog-IIR crosstalk cancellation and signal reutilization receiver for single-ended I/Os in 65 nm CMOS" VLSI, 2012.

[3] T. Toifl, et al., "A $2.6 \mathrm{~mW} / \mathrm{Gbps} 12.5$ Gbps RX With 8-Tap Switched-Capacitor DFE in 32 nm CMOS," JSSC, Apr. 2012. 